\title{
A prospective cohort study to assess the association between early pregnancy bleeding and risk of poor pregnancy outcome
}

\author{
S N Samarakkody ${ }^{\mathrm{a}}, \mathrm{K}$ Gunawardena ${ }^{\mathrm{b}}$
}

\begin{abstract}
Introduction

$16-25 \%$ of the pregnancies are complicated by threatened miscarriage. The knowledge on outcomes of the pregnancy following a threatened miscarriage is important to the obstetrician in surveillance, decision making and planning further management of the pregnancy. Therefore the need of data on the adverse maternal and perinatal outcomes following a threaten miscarriage is well evident in local context.
\end{abstract}

\section{Objectives}

To assess the association between early pregnancy bleeding and risk of poor maternal and perinatal outcomes.

\section{Methods}

A prospective cohort study was carried out in the Obstetrics and Gynaecology Unit, Teaching Hospital Peradeniya for a period of ten months. All pregnant women with a history of notable vaginal bleeding in the first 24 weeks of gestation were recruited as subjects and age and parity matched, pregnant women without a history of any vaginal bleeding, were recruited as controls. Multiple pregnancies or foetus with congenital anomalies, women with known bleeding disorders and congenital uterine abnormalities were excluded. Sample size was 440 including 220 in each group. Several selected maternal and perinatal events were considered as outcome variables. Small for gestational age was detected via locally adapted customized growth charts. Data were analysed by using SPSS 23.0. Ethical clearance was obtained by Ethics Review Committee Faculty of Medicine, Peradeniya.

\section{Results}

The mean maternal ages were 27.8 and 28.2 years respectively in study and control groups. Mean age and body mass index were comparable in the two groups. Threatened miscarriage was significantly associated with an increased relative risk for small for gestational age $(\mathrm{P}<0.01$; RR 1.52; 95\% $\mathrm{Cl}=1.10-2.08)$, low birth weight $(\mathrm{P}<0.01$; RR 1.61; $95 \% \mathrm{Cl}=1.23-2.15)$, preterm delivery $(P=0.020 ; R R$ 2.62; 95\% Cl=1.18-5.79), pre-eclampsia $(\mathrm{P}=0.04$; RR3.25; $95 \% \mathrm{Cl}=1.07-9.81)$ and foetal growth restriction $(\mathrm{P}<0.03$; $\mathrm{RR} 2.50 ; 95 \% \mathrm{Cl}=1.12-5.55)$ compared to the age and parity matched control group. There were no statistically significant difference noted between the study and control groups with regard to incidence of placental abruption, placenta praevia, retained placenta, rate of caesarean section, rate of instrumental delivery, incidence of neonatal death, Apgar score $<7$ at 5 minutes and rate of admission to neonatal intensive care unit.

\section{Conclusions and Recommendations}

Threatened miscarriage carries a significant risk of adverse maternal and perinatal outcomes and it is identified as a risk factor for small for gestational age, low birth weight, foetal growth restriction, preeclampsia and preterm labour. Placental abruption is associated with threatened miscarriage with an increased relative risk, though the association was not significant. This knowledge should be applied by the clinicians to counsel the pregnant women with threatened miscarriage and at the same time be alert on symptoms and signs of those adverse effects in order to plan the future management. Probability of inclusion of early prediction of adverse maternal and perinatal events by threatened miscarriage in to obstetric management guidelines should be determined. In future studies it is recommended to evaluate association between each adverse outcome and threatened miscarriage independently and to evaluate the outcomes following bleeding in the first and second trimesters separately.

Key words: Risk of adverse outcomes, threatened miscarriage, maternal and perinatal outcomes

Sri Lanka Journal of Obstetrics and Gynaecology 2019; 41: 8-14

DOI: http://doi.org/10.4038/sljog.v41i1.7877

${ }^{a}$ Senior Registrar in Obstetrics and Gynaecology, Professorial Unit, Colombo South Teaching Hospital, Sri Lanka

${ }^{\mathrm{b}}$ Professor of Obstetrics and Gynaecology and Chair, Department of Obstetrics and Gynaecology, Faculty of Medicine, University of Peradeniya, Sri Lanka

Correspondence: SNS, e-mail: <sumeeranirmal@yahoo.com>

Received $3^{\text {rd }}$ January 2018 and revised version accepted 30 $0^{\text {th }}$ March 2019.

http://orcid.org/0000-0003-4183-0011

Competing interest: The authors report no conflict of interest

This is an open-access article distributed under the terms of the Creative Commons Attribution 4.0 International License, which permits unrestricted use, distribution and reproduction in any medium provided the original author and source are credited. 


\section{Introduction}

The pregnancies complicated by early pregnancy bleeding with a viable foetus ${ }^{1,2}$ is known as threatened miscarriage (16-25\%). It is defined as per vaginal bleeding prior to 24 weeks of gestation, where the diagnosis is made on ultrasonic evidence of foetal cardiac activity in an intrauterine gestational sac along with a closed cervix on clinical examination ${ }^{3}$. The knowledge on outcome of the pregnancy following early pregnancy bleeding or threatened miscarriage is important to the obstetrician in surveillance, decision making and to plan further management of the pregnancy.

Threatened miscarriages have been reported as a risk factor for adverse obstetric or maternal outcomes such as small for gestational age, preterm labour, preterm pre-labour rupture of membranes, low birth weight, pregnancy induced hypertension and placental abruption. Even though, several studies have been conducted in the past looking for an association between early pregnancy bleeding and adverse perinatal outcomes, only a few studies have been conducted looking for associated maternal adverse effects. Further knowledge on the possible pregnancy outcome is useful to do a risk assessment for the rest of the pregnancy and to offer a suitable management plan. This will help in reducing poor maternal and foetal outcomes.

Early identification and detection of the risk factors for poor maternal and perinatal outcome is of utmost importance and is a more feasible option to reduce the complication and improve neonatal morbidity and mortality. For this we need research based evidence to assess the relationship between early pregnancy bleeding and related adverse maternal and perinatal outcomes as Saraswat et al has suggested in their systematic meta-analysis that more prospective cohort studies should be conducted ${ }^{4}$.

Only a few studies are reported in literature describing the association between early pregnancy bleeding or threatened miscarriage and adverse pregnancy outcomes. Currently threatened miscarriage is not considered as a risk factor in relation to adverse maternal and perinatal outcomes in pregnancy, apart for the specific period of active bleeding 5 .

No studies have been reported on the association of early pregnancy bleeding and SGA in a Sri Lankan setting, using the newly validated local reference ${ }^{6}$ levels for SGA. This will be the first study to describe the association using these values. Therefore, we hope to carry out a cohort study on maternal and perinatal outcomes following threatened miscarriage among a group of pregnant women attending antenatal clinics at a tertiary care hospital.

\section{Methodology}

This was a prospective cohort study carried out at the antenatal clinics (all five of them) and professorial obstetrics and gynaecology wards of the Teaching Hospital Peradeniya. Study population was pregnant women with singleton pregnancies between 10-24 weeks of gestation with a history of notable vaginal bleeding in the first twenty four weeks of gestation. They were recruited as subjects and age and parity matched, pregnant women without a history of any vaginal bleeding, were recruited as controls. Recruitment was done over a period of 10 months from August 2016 to May 2017. They were prospectively followed up till delivery. Women having large fibroid uterus distorting the uterine cavity, carrying multiple pregnancies, foetus with congenital anomalies, those with known thrombophilia/bleeding diathesis, having congenital uterine abnormalities and with previous history of preterm labour or small for gestational age baby were excluded.

The primary outcome measured was 'Small for Gestational Age (SGA)' which was defined as foetal weight estimated by ultrasound examination of less than tenth percentile or birth weight of less than tenth percentile for gestational age according to the local reference values ${ }^{6,7}$.

The secondary outcomes measured for adverse maternal outcomes were Preterm labour, PPROM, Preeclampsia, Placental abruption (premature, partial or complete separation of the placenta), Placenta praevia (placenta in the lower uterine segment, completely or partially covering the internal os), incidence of retained placenta that might be associated with early pregnancy bleeding among women who carry their pregnancy to foetal viability.

Adverse perinatal outcomes or neonatal complications were measured as incidence of foetal growth restriction, neonatal deaths, low 5 minute Apgar scores $(<7)$, rate of admission to the NICU that may be associated with early pregnancy bleeding among women who deliver beyond gestation of foetal viability.

\section{Procedure:}

All participants in the study were subjected to routine history taking with a general and obstetric examination. 
A pelvic Cusco bivalve self-retaining speculum was carried out to confirm bleeding through the closed cervix only in the study population.

Data collection was carried out using an interviewer administered questionnaire, and each participant in the study group underwent a trans-abdominal ultrasound scan to diagnose the threatened miscarriage, done by the principal investigator in order to avoid inter-observer variability.

Small for gestational age was assessed with ultrasound scan derived estimated weight of the foetus at a given time between the period of gestation 24 weeks to 41 weeks. The estimated foetal weight was plotted against a validated, locally adapted estimated foetal weight centile chart $^{6}$ for the Sri Lankan population in which the estimated foetal weight of less than tenth percentile for the appropriate gestational age was defined as small for gestational age. Foetal growth restriction was assessed by serial growth scans from 24 weeks on wards 3 weekly apart in order to detect the growth velocity and plotted against the customized centile charts.

The sample size calculation was done using the WinPepi software. This was done to assess the association between early pregnancy bleeding and SGA using data from the study by Arafa et al. 193 in each group were required. Since we did not have data on the possible rate of lost to follow up, it was assumed to be close to $15 \%$ due to delivery in other centres. Therefore, 220 women were recruited to the two groups of subjects and controls at the recruitment.

Mean and Standard Deviations values were used to describe continuous variables. Percentages and Frequency were used to describe categorical variables. For risk estimation, relative risk was calculated. Probability cut off value was 0.05 and $95 \%$ confidence interval was used for statistical significance. The associations between exposure and adverse outcomes were assessed using chi square test and Fishers-extract test for smaller numbers. Relative rate calculation was done for all outcome variables and a significance level of 5\% was used in the analysis. The data analysis was done using the Statistical Package for the Social Sciences (SPSS) version 23.0. Categorical variables were described by percentages and frequencies. Numerical variables were described by measures of central tendency.

Informed and written consents were gained from all study participants and they had the right to withdraw from the follow up without any consequences to their clinical management. Participant identification data wasn't recorded to maintain confidentiality. Ethical approval for the study was taken from the ethical review committee of the Faculty of Medicine, University of Peradeniya and the relevant permissions were obtained from the director of the Teaching Hospital Peradeniya and the lead clinicians.

\section{Results}

Study sample consisted of 440 study participants who included 220 mothers with threatened miscarriage and 220 controls (Table 1 ).

There is homogeneity among the study participants in terms of their baseline characteristics in both arms.

The women in the study group with threatened miscarriage were more likely to suffer from small for gestational age (RR $1.52,95 \%$ CI 1.10-2.08) and the findings were statistically significant $(\mathrm{P}<0.01)$ in the study group compared to the control group. Similar significance $(\mathrm{P}<0.01)$ was detected for the low birth weight (birth weight less than $2500 \mathrm{~g}$ ) neonates born to the women with threatened miscarriage with a relative risk of 1.61 (95\% CI 1.23-2.15) (Table 2).

As shown in Table 2 there were statistically significant increased incidence of preterm delivery in the study group than the controlled group $(\mathrm{P}=0.020)$ and also those with threatened miscarriage were more prone to develop pre-eclampsia $(\mathrm{P}=0.045)$.

In those with early pregnancy bleeding even though there was an increase incidence of placenta praevia $(0.9 \%)$ and placental abruption $(2.7 \%)$ in the study group than the control $(0.5 \%$ and $1.4 \%$ respectively) the difference didn't reach statistical significance and when it came to retained placenta the incidence was higher $(1.4 \%)$, however no cases were reported in the control group.

The caesarean section rates and the rates of instrumental delivery in both the study and the control group were similar and held no statistical significance (Table 3).

There was a statistically significant increase in the incidence of foetal growth restriction $(\mathrm{P}=0.030)$ in the study group with a relative risk of $2.50(95 \%$ $\mathrm{CI}=1.12-5.55)$ in threatened miscarriage group than the control group. 
Table 1. Distribution of characteristics of the participants

\begin{tabular}{|l|l|l|}
\hline Characteristics & Study group & Control group \\
\hline Mean maternal age & $27.8( \pm 7.2)$ & $28.2( \pm 6.8)$ \\
Maternal age range & $18-41$ & $18-41$ \\
Married [n (\%)] & $218(99)$ & $217(98.6)$ \\
Maternal BMI at booking & $23.8( \pm 2.9)$ & $23.0( \pm 3.3)$ \\
Period of amenorrhoea at booking (weeks) & 9.1 & 8.9 \\
Period of amenorrhoea at delivery (weeks) & $38.40( \pm 2.07)$ & $39.18( \pm 1.32)$ \\
\hline
\end{tabular}

Data are expressed as mean $( \pm$ standard deviation); or n $(\%)$; BMI $=$ Body Mass Index

Table 2. Adverse maternal outcomes in pregnant women with threatened miscarriage

\begin{tabular}{|c|c|c|c|c|c|c|}
\hline $\begin{array}{l}\text { Adverse } \\
\text { maternal } \\
\text { outcome }\end{array}$ & $\begin{array}{l}\text { Threatened } \\
\text { miscarriage } \\
(n=220)\end{array}$ & $\%$ & $\begin{array}{l}\text { Control } \\
(n=220)\end{array}$ & $\%$ & Probability & $\begin{array}{c}\text { Relative } \\
\text { risk } \\
(95 \% \mathrm{CI})\end{array}$ \\
\hline Preterm labour & 21 & 9.5 & 8 & 3.6 & 0.020 & $\begin{array}{l}2.62 \\
(1.18-5.79)\end{array}$ \\
\hline PPROM & 27 & 12.3 & 23 & 10.5 & 0.653 & $\begin{array}{l}1.17 \\
(0.69-1.98)\end{array}$ \\
\hline Preeclampsia & 13 & 5.9 & 4 & 1.8 & 0.045 & $\begin{array}{l}3.25 \\
(1.07-9.81)\end{array}$ \\
\hline Placental abruption & 6 & 2.7 & 3 & 1.4 & 0.503 & $\begin{array}{l}2.00 \\
(0.51-7.89)\end{array}$ \\
\hline Placenta praevia & 2 & 0.9 & 1 & 0.5 & 1.000 & \\
\hline Retained placenta & 3 & 1.4 & 0 & 0 & - & \\
\hline Delivery via LSCS & 41 & 18.6 & 53 & 24.1 & 0.201 & $\begin{array}{l}0.774 \\
(0.54-1.11)\end{array}$ \\
\hline Instrumental Delivery & 9 & 4.1 & 10 & 4.5 & 1.000 & $\begin{array}{l}0.900 \\
(0.37-2.17)\end{array}$ \\
\hline
\end{tabular}

$\mathrm{CI}=$ Confidence Interval; PPROM = Preterm Prelabour Rupture of Membranes; LSCS = Lower Segment Caesarean Section. 
Table 3. Adverse perinatal outcomes in pregnant women with threatened miscarriage

\begin{tabular}{|l|c|c|c|c|c|c|}
\hline $\begin{array}{l}\text { Adverse } \\
\text { perinatal } \\
\text { outcome }\end{array}$ & $\begin{array}{c}\text { Threatened } \\
\text { miscarriage } \\
(\mathrm{n}=220)\end{array}$ & $\%$ & $\begin{array}{c}\text { Control } \\
(\mathrm{n}=220)\end{array}$ & $\%$ & Probability & $\begin{array}{c}\text { Relative } \\
\text { risk } \\
(95 \% \text { CI })\end{array}$ \\
\hline FGR & 20 & 9.1 & 8 & 3.6 & 0.030 & $\begin{array}{l}2.50 \\
1.12-5.55 \\
\text { Neonatal Death }\end{array}$ \\
Apgar at 5 minutes $<7$ & 6 & 1.4 & 1 & 0.5 & 0.623 & $\begin{array}{l}2.62 \\
(0.31-28.6) \\
\text { Admission to NICU }\end{array}$ \\
\hline
\end{tabular}

FGR $=$ Foetal Growth Restriction; NICU $=$ Neonatal Intensive Care Unit.

There were no statistically significant differences observed in the two groups in neonatal death rate, Apgar score $<7$ at 5 minutes and rate of admission to neonatal intensive care unit.

\section{Discussion}

In our study we have identified 13 different maternal and perinatal outcomes and have found several associations between early pregnancy bleeding and the poor pregnancy outcomes.

The results of the study clearly demonstrated that small for gestational age, low birth weight (birth weight less than $2500 \mathrm{~g}$ ), foetal growth restriction, preeclampsia and preterm labour are significantly associated with the pregnant women with threatened miscarriage with a higher relative risk.

Although the incidences of preterm prelabour rupture of membranes and placental abruption were not statistically significant in the threatened miscarriage group, both the events were found to have high relative risks. The significance of retained placenta in the threatened miscarriage group could not be compared as no cases of retained placentae were reported in the control group.

In assessing perinatal adverse effects, though no significant risk was observed in the study group for Apgar score $<7$ at 5 minutes and rate of admission to neonatal intensive care unit, a higher relative risk was found in the rate of admission to neonatal intensive care unit in the neonates born to pregnant women within threatened miscarriage group.

To our knowledge, this is the only study conducted in Sri Lanka, to assess the risk of small for gestational age associated with threatened miscarriage, where the diagnosis of small for gestational age has been made with ultrasound assessments plotted in the locally adapted customized growth charts. This enhances the validity of the study findings to the local pregnant population.

Weiss et $\mathrm{al}^{8}$ and Patel et $\mathrm{al}^{9}$ reported statistically significant increase incidence of intra uterine growth restriction and placental abruption in the women with threatened miscarriage. The incidence of small for gestational age was significantly higher in the study group which again supported the association of foetal growth restriction and the threatened miscarriage.

Our study revealed a significant association between preterm delivery (delivery < 37 completed weeks) and threatened miscarriage, in line with the findings from previous researches ${ }^{2,5,10}$. In a local study conducted at the Sri Jayawardenapura Hospital found a significant association between preterm delivery and the early pregnancy bleeding within the first 14 weeks of gestation. It can be assumed that the preterm labour might be precipitated by an inflammatory reaction 
caused by the presence of blood or a blood clot following a threatened miscarriage, in between the placenta and the amnion (chorioamniotic space) disrupting the space. The resultant hematoma could also act as a focus for intrauterine infection that may stimulate contractions in the uterine myometrium.

Though the incidence of preterm pre-labour rupture of membrane was not prominent in the present study, a few studies ${ }^{11,12}$ reported that increase incidence of preterm pre-labour rupture of membrane is associated with threatened miscarriage.

Threatened miscarriage as a predictor of adverse maternal and perinatal outcomes such as small for gestational age, foetal growth restriction, pre-term labour and pre eclampsia, will be helpful in prevention of complications by close observation, monitoring of high risk groups and follow up of mothers with early pregnancy bleeding. Identification of high risk women would be important to implement treatment modalities (e.g.: progesterone) early in the gestation to prevent the occurrence of pre-term delivery that would have lead to a health burden in many aspects as well as psychological stress to the mother.

Highest number of unexplained foetal death in utero is said to be due to undiagnosed or undetected foetal growth restriction or small for gestational age. Therefore it is important to detect those vulnerable foetuses early in the antenatal period to prevent adverse outcomes.

It is again vital to have a high suspicion and lower threshold in diagnosing pre eclampsia in pregnant women with a history of threatened abortion, if they present with suggestive symptoms and signs.

Even though majority of the pregnant women with threatened miscarriage carries a good prognosis in their pregnancy outcomes similar to the mothers who are not found to have bleeding in their early pregnancy, it is noticeably evident that those mothers have an increase relative risk of some maternal and perinatal adverse outcomes.

Our study included pregnant mothers attended to a single unit or hospital as the study population. We suggest that if the study was conducted in a multicentre setting, it would have enhanced the external validity of the study further. Furthermore the amount of bleeding in the study group was not quantified or assessed clinically in the present study. In here is a possibility to assess the adverse maternal and perinatal outcomes against the degree of bleeding. Also, the effects of anaemia caused by threatened miscarriage could be evaluated.

Additionally an expert in the radiology field could be recruited to collect ultrasound measurements in small for gestational age and foetal growth restriction parameters (estimated foetal weight) assessment and measures can be taken to minimise measurement bias.

\section{Limitations}

Measurement bias and selection bias as the principal investigator himself performed the Ultrasound scan and also got directly involved in the clinical management of the study participants. Study participants were from different age groups and the parity of them weren't the same.

Uniformity of the factors related to the exposure variables among the study participants was low.

The financial constraints and the limitation of the time frame of the study, made it difficult to alter the methodology in order to minimize above effects.

\section{Conclusions and recommendations}

\section{Conclusion}

Threatened miscarriage carries a significant risk of adverse maternal and perinatal outcomes compared to the pregnancies not complicated by threatened miscarriage.

Threatened miscarriage is identified as a risk factor for small for gestational age, low birth weight, foetal growth restriction, preeclampsia and preterm labour. However, placental abruption is associated with threatened miscarriage with an increased relative risk, though the association was not significant.

\section{Recommendation}

Association between threatened miscarriage and each maternal and perinatal outcome should be evaluated independently in different studies. It would be best if the outcomes following bleeding in the first and second trimesters were described separately.

Clinicians should be well aware about the associations and risks related to threatened miscarriage. This 
knowledge should be applied by the clinicians to counsel the pregnant women with threatened miscarriage and at the same time to be alert on symptoms and signs of those adverse effects in order to plan the future management.

Probability of inclusion of early prediction of adverse maternal and perinatal events by threatened miscarriage in to obstetric management guidelines should be determined.

\section{References}

1. Mulik V, Bethel J, Bhal K. A retrospective population based study of primi gravid women on the potential effects of threatened miscarriage on obstetric outcome. British Journal of Obstetrics and Gynaecology 2004 April; 24(3): 249-53.

2. Farrell T, Owen P. The significance of extra chorionic membrane separation in threatened miscarriage. British Journal of Obstetrics and Gynaecology 1996; 103: 926-28.

3. Cunningham FG, Gant NF, Leveno KJ, Gilstrap LC, Hauth JC, Wenstrom KD, editors. William's Obstetrics. $21^{\text {st }}$ edition. New York (NY): McGrawHill; 2001. Chapter 6.

4. Saraswat L, Bhattacharya S, Maheshwari A. Maternal and perinatal outcome in women with threatened miscarriage in the first trimester: a systematic review. British Journal of Obstetrics and Gynaecology 2009; 117(3): 245-57.

5. Batzofin JH, Fielding WL, Friedman EA. Effect of vaginal bleeding in early pregnancy on outcome. Obstetrics and Gynecology 1984; 63: 515-18.
6. Dias T, Shanmugaraja V, Ganeshamoorthy, Kumarasiri S, Abeykoon S, Padeniya T. Birth weight standards - Ability of birth weight percentiles in predicting abnormal fetal growth and outcome. Sri Lanka Journal of Obstetrics and Gynaecology 2014 Dec; 36: 85-8.

7. Kumarasiri S, Wanigasekara R, Wahalawatta L, Jayasinghe L, Padeniya T, Dias T. Accuracy of ultrasound estimated fetal weight formulae to predict actual birth weight after 34 weeks: prospective validation study. Ceylon Medical Journal 2013 Sep; 58(3): 116-21.

8. Weiss JL, Malone FD, Vidaver J, Ball RH, Nyberg DA, Comstock CH. Threatened abortion: a risk factor for poor pregnancy outcome, a populationbased screening study. American Journal of Obstetrics and Gynecology 2004; 190: 745-50.

9. Patel BI, Trivedi V. Threatened abortion outcome in relation to intrauterine clot site and not only volume. International Journal of Gynecology and Obstetrics 2000; 70(4): D44.

10. Williams MA, Mittendorf R, Lieberman E, Monson RR. Adverse infant outcomes associated with firsttrimester vaginal bleeding. Obstetrics and Gynecology 1991; 78: 14-18.

11. Funderburk SJ, Guthrie D, Meldrum D. Outcome of pregnancies complicated by early vaginal bleeding. British Journal of Obstetrics and Gynaecology 1980; 87: 100-5.

12. Das AG, Gopalan S, Dhaliwal LK. Fetal growth and perinatal outcome of pregnancies continuing after threatened abortion. Aust N Z J Obstet Gynaecol. 1996 May; 36(2): 135-39. 